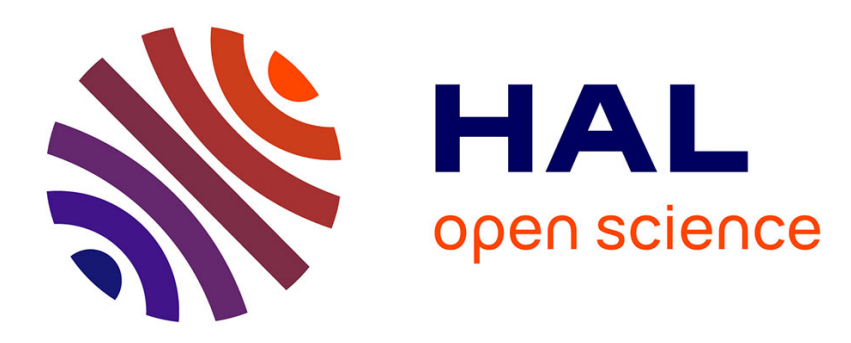

\title{
Review of superconducting magnetometers and cryogenic refrigeration techniques \\ W.S. Goree
}

\section{To cite this version:}

W.S. Goree. Review of superconducting magnetometers and cryogenic refrigeration techniques. Revue de Physique Appliquée, 1970, 5 (1), pp.3-12. 10.1051/rphysap:01970005010300 . jpa-00243372

HAL Id: jpa-00243372

https://hal.science/jpa-00243372

Submitted on 1 Jan 1970

HAL is a multi-disciplinary open access archive for the deposit and dissemination of scientific research documents, whether they are published or not. The documents may come from teaching and research institutions in France or abroad, or from public or private research centers.
L'archive ouverte pluridisciplinaire HAL, est destinée au dépôt et à la diffusion de documents scientifiques de niveau recherche, publiés ou non, émanant des établissements d'enseignement et de recherche français ou étrangers, des laboratoires publics ou privés. 


\title{
REVIEW OF SUPERCONDUGTING MAGNETOMETERS AND GRYOGENIG REFRIGERATION TEGHNIQUES
}

\author{
By W. S. GOREE, \\ Stanford Research Institute, Low Temperature Physics Department, Menlo Park, California (U.S.A.) (1).
}

\begin{abstract}
Superconductors are characterized by many unique properties which are all manifestations of the macroscopic quantum nature of the superconducting state. Several of these properties háve direct application to devices for shielding, stabilizing, measuring and producing magnetic fields. The zero resistance property of superconductors has been used to construct magnets capable of producing magnetic fields up to 140 kgauss. This same property also provides for excellent magnetic shielding, i.e., the net magnetic flux linking a closed superconducting path must remain constant as long as the path remains superconducting. The somewhat more subtle property of quantized magnetic flux has been used in the construction of sensitive magnetometers with field resolution as good as $10^{-7}$ gauss.

Tunneling of superconducting electron pairs through a non-superconducting barrier was predicted by Josephson in 1962 and verified experimentally the following year by Rowell. Various extensions of the Josephson tunneling properties of superconductors have remarkable device applications. In particulat the tunneling characteristics are changed appreciably by external magnetic fields. Magnetometers using this property have been used to resolve field changes as small as $10^{-10}$ gauss.

The application of these properties of superconductivity, especially to sensitive magnetometry, will be reviewed to lay the groundwork for the more detailed papers to follow in this session.

All of the devices noted above require temperatures near $4 \mathrm{oK}$ in order to function. This is a particularly significant requirement for space instruments and for many earth-based geophysical measurements as well. Liquid helium refill systems are the simplest cooling techniques, although serious logistic and handling problems may be encountered in field applications. Closed cycle miniature refrigerators could make superconducting magnetometers, shields, etc., practical field instruments. The present state of the art in all of these cooling techniques will be reviewed.
\end{abstract}

In the first part of this paper, I will review the properties of superconductivity that have been used in the construction of very sensitive magnetometers, the purpose being both to present an overall view of the ways superconductivity has been used and to compare the various techniques with one another and with more conventional magnetometers. I will briefly explain the general principles of operation of these magnetometers and give some idea of the circuit configurations that have been used. Other papers in this session will go into far more detail on specific instruments and the performance, construction, and limitations of these instruments. The second part of the paper will be a review of cooling techniques applicable for superconducting circuits.

Magnetometers. - There are basically two types of superconducting magnetometers that have been studied. Both types are vector magnetometers with field resolutions ranging from $10^{-5}$ to $\sim 7 \times 10^{-11}$ gauss. The first type we will call flux motion magnetometers. In these magnetometers various properties of the super-

(1) Present Address : DEVELCO INC., Mountain View Calif. (U.S.A.). conducting state are used to move ambient magnetic flux relative to a pickup coil. This flux movement induces an electromotive force in the coil that is a function of the ambient magnetic field or of a field change. The properties that have been used in flux motion magnetometers are zero resistance, the Meissner effect [1], quantized flux [2] and flux flow [3].

The second type of superconducting magnetometer depends on the tunneling characteristics of superconductors [4]. If two superconductors are separated by a nonsuperconducting barrier, we find that under the proper conditions currents can flow through the barrier without a voltage being developed. A critical current exists, though, whose magnitude is a function of the gauge invariant phase difference of the superconducting wave function across the barrier. This phase difference is determined by the transport current flowing through the junction and the magnetic field at the junction. It is the variation of the critical current with the phase or consequently with the external magnetic field that is used to measure changes in the magnetic field.

Now let us return to the flux motion type devices and discuss some of the specific circuits that have been developed for magnetometers. The Meissner effect 
magnetometer was the first and probably the simplest, at least conceptually, superconductor magnetometer that has been constructed [5]. Its general operating characteristics are basically the same as for any flux motion type device.

If a superconducting post is inserted into a pickup coil located in an axial magnetic field, as shown in figure 1 , the field will be pushed out of the way causing a change in the flux linking the coil. This

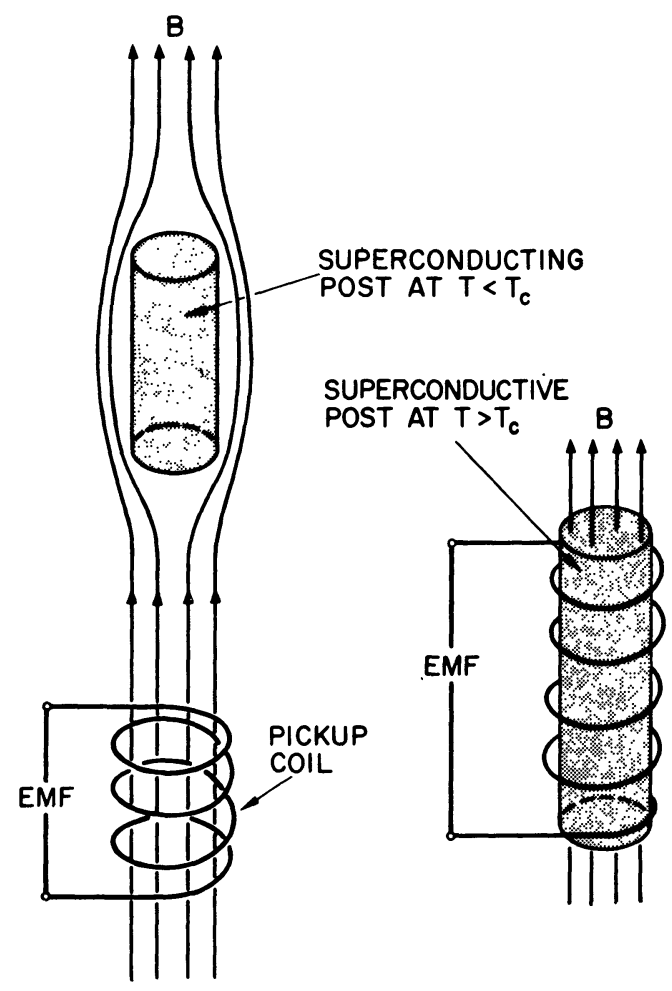

FIG. 1. - Meissner type magnetometer.

flux change induces an EMF in the coil that is proportional to the ambient field. Now instead of physically moving the superconducting post we can heat and cool it above and below its critical temperature, $T_{\mathrm{c}}$. In a finite magnetic field, currents are spontaneously induced at the critical temperature to expel the magnetic field from the superconducting material. This flux expulsion is called the Meissner effect. The same flux change occurs as when the post is inserted into the pickup coil and the resultant EMF is of course proportional to the magnetic flux expelled from the body of the sample. If the switching (heating and cooling) is done rapidly an alternating voltage will be induced in the coil whose amplitude will be linearly proportional to the absolute magnetic field at the sample in the direction of the pickup coil axis. A typical sample-heater assembly is shown in figure 2 . Meissner modulators of this type have been switched at frequencies up to $30 \mathrm{kHz}$ with field resolution as good as $10^{-6}$ gauss.

The quantized flux property of superconductivity provides an even more sensitive means for magnetic field measurement. This property, predicted by Fritz London in 1948 [6] and verified experimentally by Deaver and Fairbank [7] and Doll and Naubauer [8] in 1961 , states that the canonical momentum of

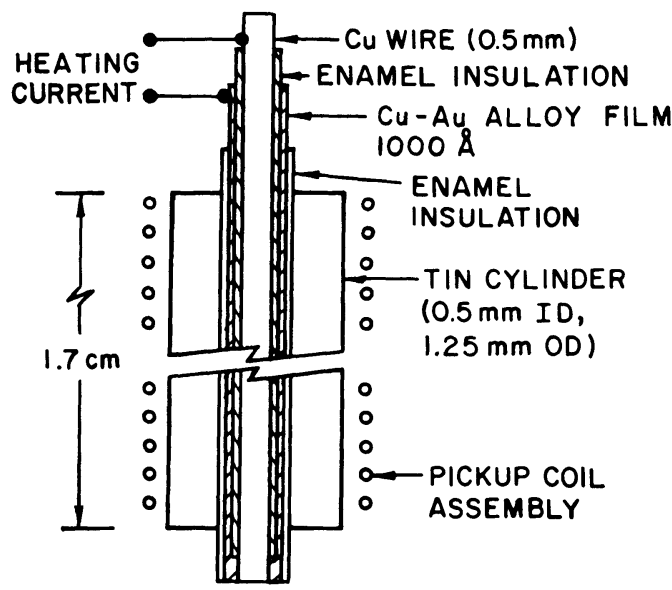

FIG. 2. - Meissner modulator construction.

superconducting electrons integrated around a closed path in a superconductor is quantitized, i.e. :

$$
\oint\left(2 m \mathbf{V}+\frac{2 e}{c} \mathbf{A}\right) \cdot \mathrm{d} \mathbf{l}=n h .
$$

This means that if a superconducting cylinder is cooled below its critical temperature, $T_{\mathrm{c}}$, in a magnetic field producing nonintegral flux quantum (the flux quantum, $\varphi_{0}$, is $2.07 \times 10^{-7}$ gauss $/ \mathrm{cm}^{2}$ ) through the cylinder, then currents are induced in the walls of the cylinder to change the flux to the nearest integral multiple of $\varphi_{0}, n \varphi_{0}$. The currents induce an EMF proportional to the difference between $n \varphi_{0}$ and the initial flux. This property is used as a magnetometer in almost the identical fashion of the hollow Meissner modulator described earlier.

The response of this modulator to magnetic fields will be as shown in figure 3 . We can see a linear section between quantized flux steps and the abrupt change of sign of the voltage at half interval values of the flux quantum. One possible feature of this type of magnetometer is a digital response as a function of magnetic field. In general, the quantized flux modulator will have walls thick enough so that it will also exhibit a Meissner effect. In this case the

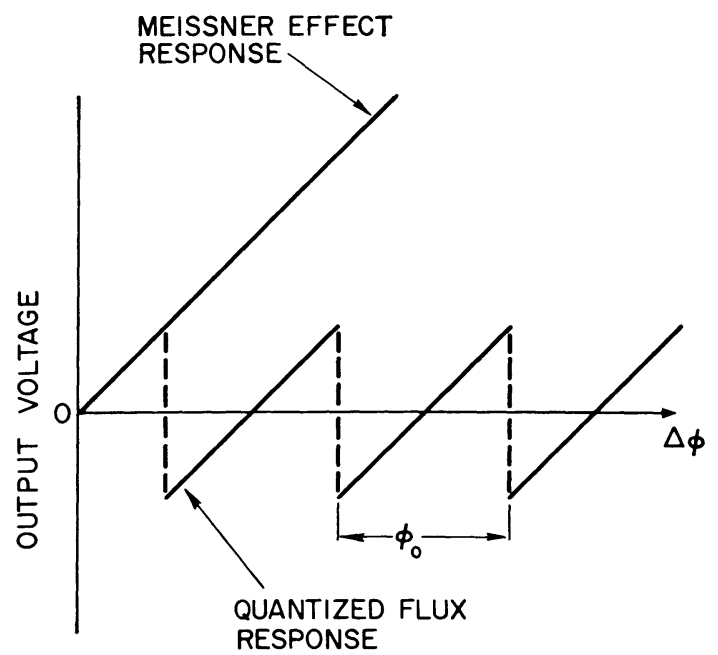

FIG. 3. - Response of Meissner effect and quantized flux magnetometers.

Erratum : In this figure and others, subscript $ø$ means $\varphi$. 
response will be a combination of the periodic quantized flux response and the linear Meissner response also as shown in figure 3. Magnetic field resolutions as good as $10^{-7}$ gauss have been obtained with quantized flux magnetometers [5].

The significant differences between the quantized flux and Meissner modulators are :

1. The switching frequencies can be as high as several megahertz with the quantized flux circuit, compared with tens of kilohertz for the Meissner circuit.

2. The response of the quantized flux circuit as a function of magnetic field is periodic in the flux quantum.

3. The quantized flux modulator is generally very small : $20 \mu$ o.d. with $1 \mu$ wall thickness.

The flux flow magnetometer [3] of Mercereau and Nisenoff uses the properties of a critical current and fluxoid quantization. In practice their circuit is very similar to a quantized flux modulator. A thin film of superconducting material is deposited on a small dielectric cylinder, usually a millimeter or so o.d. by one centimeter long. A small bridge as shown on figure 4 is photoetched in the cylinder. The detail

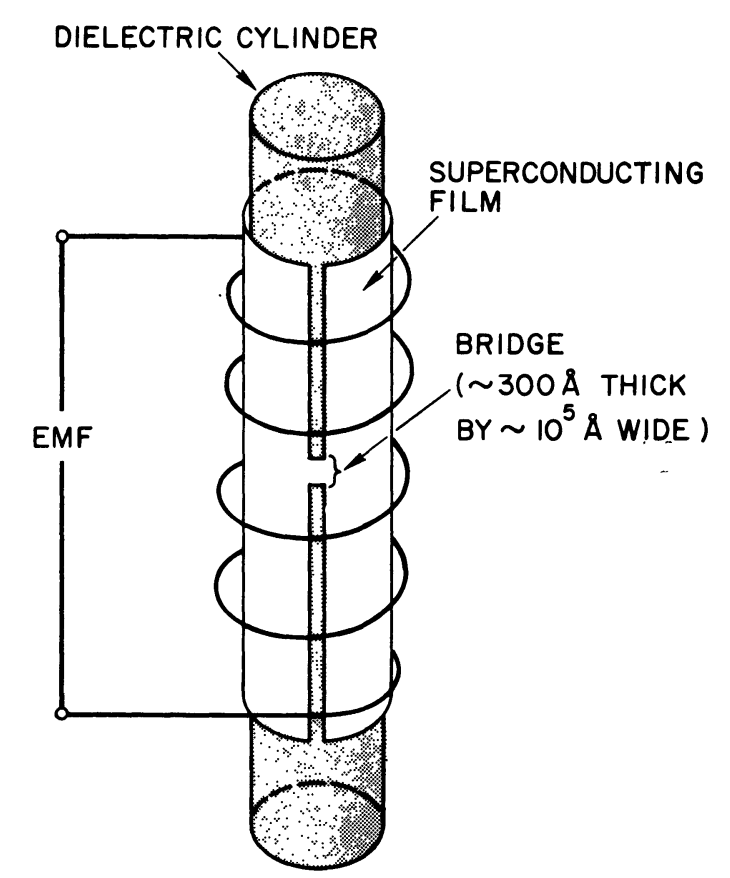

FIG. 4. - Mercereau-Nisenoff magnetometer.

operation of this magnetometer will be presented by Mercereau and Nisenoff in the next two papers. Magnetic field resolution as good as $10^{-10}$ gauss has been obtained with this circuit.

Zero resistance alone is the crucial superconducting property used in an elegant magnetometer being developed by Fairbank and Opfer, to be discussed later this afternoon. A sketch of this circuit is given in figure 5 . In operation a superconducting ground plane is vibrated very rapidly near a superconducting pickup coil. Basically the vibrating plane modulates the inductance of the pickup coil in the same way

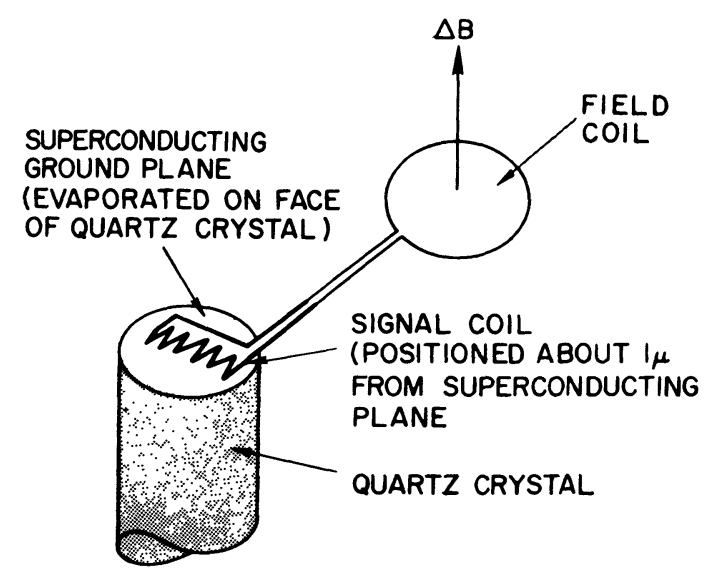

FIG. 5. - Fairbank-Opfer magnetometer.

as with the other modulators discussed earlier. In the Fairbank-Opfer circuit the ground plane is evaporated on the surface of a quartz crystal which is driven electrically at frequencies from 0.1 to $1 \mathrm{MHz}$. One significant feature of this magnetometer is that none of the superconducting components are switched out of the completely superconducting state. Also the response is linear with magnetic field. Thus, the noise due to eddy currents, flux flow, temperature change, etc., should be eliminated.

We will now consider magnetometers utilizing the tunneling properties of superconductivity. We mentioned earlier that the current density of a single Josephson Junction is a function of the phase difference of the superconducting electrons across the tunneling barrier, i.e., $j=j_{0} \sin \varphi$. We can show further that by substituting the appropriate expression for the phase in the above equation that the critical transport current through a single junction is given by an equation analogous to an optical diffraction equation for a single slit $[4,8]$ :

$$
I_{\text {crit }}=I_{0} \frac{\sin \frac{\pi \varphi_{J}}{\varphi_{0}}}{\pi \frac{\varphi_{J}}{\varphi_{0}}}
$$

where $I_{0}=$ maximum current that can flow through the junction at zero magnetic field, without producing a voltage across the junction,

$\varphi_{J}=$ magnetic flux linking the junction area, $\varphi_{0}=$ flux quantum $\simeq 2 \times 10^{-7}$ gauss $/ \mathrm{cm}^{2}$.

The critical current of a single junction is thus a periodic function of the magnetic flux linking the junction, $\varphi_{J}$, as shown in figure 6 a. The junction area is very small though, and the resultant magnetic field resolution is generally of the order of milligauss.

A far more sensitive magnetometer may be constructed if two junctions are connected in parallel, as shown in figure $6 \mathrm{~b}$. In this circuit superconducting electrons can flow from one side of the device to the other through either of the two paths, and interference can result from phase changes along these paths. This interference produces an additional modulation of the 

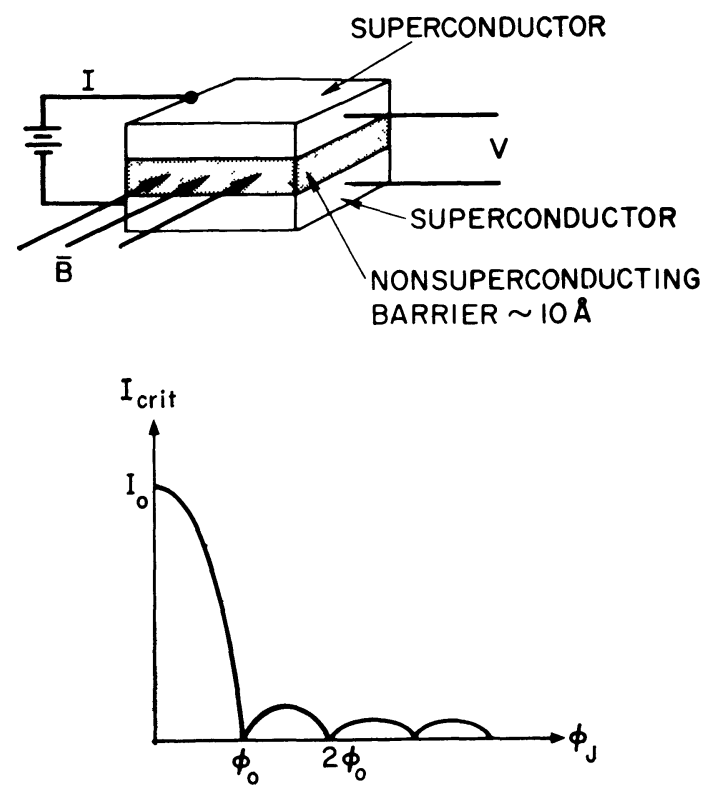

FIG. 6 a. - Single Josephson junction.

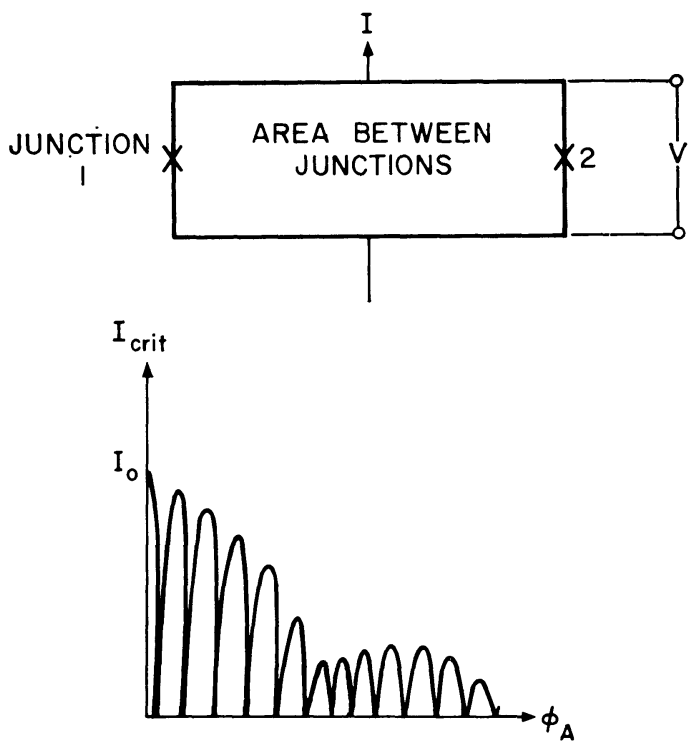

FIG. 6 b. - Double Josephson junction.

critical current of the parallel combination of junctions as given by an equation analogous to an interference equation for a two slit optical interferometer :

$$
I_{\mathrm{crit}}=I_{0} \frac{\sin \frac{\pi \varphi_{J}}{\varphi_{0}}}{\pi \frac{\varphi_{J}}{\varphi_{0}}} \cos \frac{\pi \varphi_{A}}{\varphi_{0}}
$$

where $\varphi_{A}$ is the magnetic flux linking the circuit that connects the two junctions and the critical current variation with magnetic flux is given on figure $6 \mathrm{~b}$.

Additional parallel junctions give a field response analogous to a multiple slit interferometer. De Bruyn Ouboter will discuss the behavior of interferometers containing up to six point contacts in a paper to be presented later in this conference.

Since the area of the circuit connecting the Josephson junctions may be orders of magnitude larger than the area of the junctions, the internal flux may modulate the critical current very rapidly compared with the diffraction effect of the individual junctions.

Thus, it is the fact that magnetic fields can couple to the phase of the superconducting electron pairs and thereby cause a significant change in the junction critical current that makes these devices useful as sensitive magnetometers. A plot of the double junction equation is given in figure $6 \mathrm{~b}$. We should note on this figure that the interference equation and the plot are functions of the internal magnetic flux, $\varphi_{A}$, that is, the actual flux that links the circuit connecting the two junctions $[9,10]$; the laboratory parameter is of course the external magnetic flux, $\varphi_{\text {ext }}$. Thus the measured critical current, $I_{\text {crit }}$, as a function of applied field may be very different from that shown on the figure. This difference is due to the difference between the internal and the external flux and is caused by shielding currents that are induced by the external flux to keep the internal flux constant at the value it had when the circuit was cooled below the critical temperature. This is the zero resistance property of superconductors. The fact that the internal flux changes at all means that the shielding is less than perfect. That is, magnetic flux penetrates the circuit at the junctions and causes the phase change that alters the critical current. The relationship between the external and internal flux is given by the following equation :

$$
\varphi_{\mathrm{ext}}=\varphi_{A}-L I_{0} \sin \frac{2 e}{\hbar}\left(\varphi_{A}-k \varphi_{0}\right) .
$$

This equation has been solved numerically and the resulting plot is shown on figure 7 . From this solution we find that the internal flux does not vary smoothly
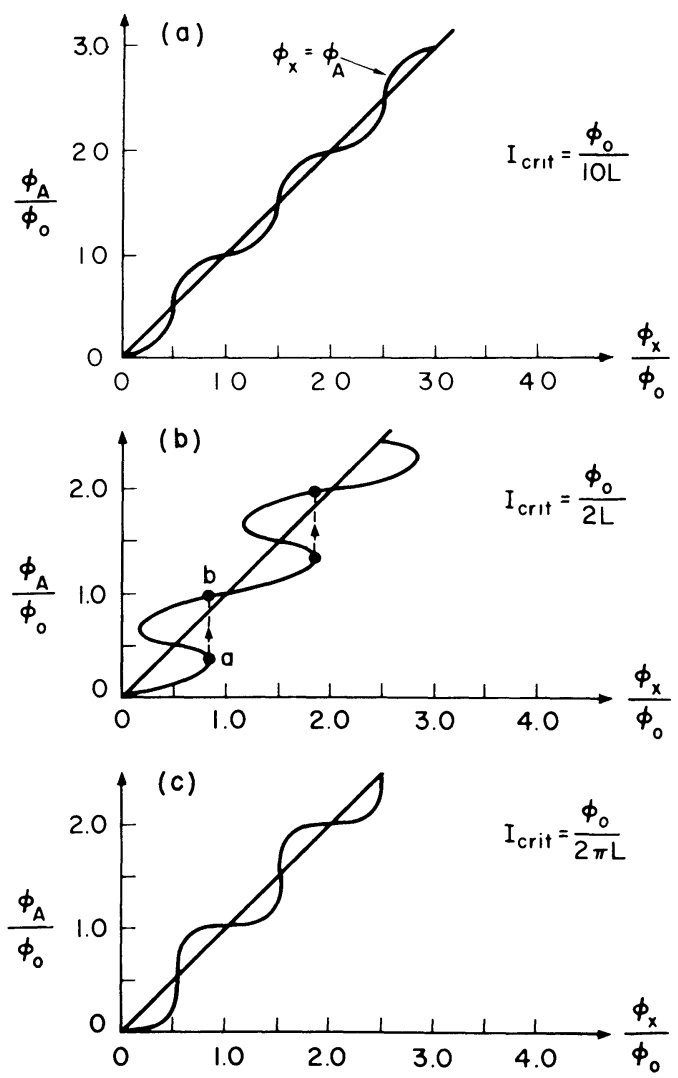

FIG. 7. - Internal versus external magnetic flux for a superconducting interferometer. 
or continuously with external flux for all values of the zero field critical current $I_{0}$ and the circuit inductance $L$. If :

$$
I_{0} \leqslant \frac{\varphi_{0}}{2 \pi L}
$$

we find complete modulation, or a smooth and single valued dependence of internal on external flux as shown on figure $7 \mathrm{a}$. If the critical current, $I_{0}$, on the other hand is greater than $\varphi_{0} /(2 \pi L)$ we find that the internal flux varies discontinuously with the external flux, but is still periodic in the flux quantum $\varphi_{0}$ $[9,10]$ as shown on figure $7 \mathrm{~b}$. The case for maximum modulation of the critical current is for $I_{\text {crit }}$ equal to $\varphi_{0} /(2 \pi L)$ as shown on figure $7 \mathrm{c}$.

This shielding is observed experimentally as an incomplete modulation of the critical current with external flux and, in effect, limits improvement of the resolution of the device that may be obtained by making the area and hence the inductance larger.

The general characteristics discussed above for tunnel junctions apply to double - and single - junction interferometers whether the junctions are evaporated film Josephson junctions [4], point contacts [10], or Dayem bridges [11]. The only qualitative difference is that the Josephson equation $j=j_{0} \sin \varphi$ may not hold exactly for different junctions, thereby changing the specific relation between the critical current, flux unit, and the inductance.

Many different types of interferometers have been constructed from the various superconducting junctions shown on figure 8 . The single point contact device [10] is very similar to the magnetometer to be discussed by Mercereau and Nisenoff. John Clarke

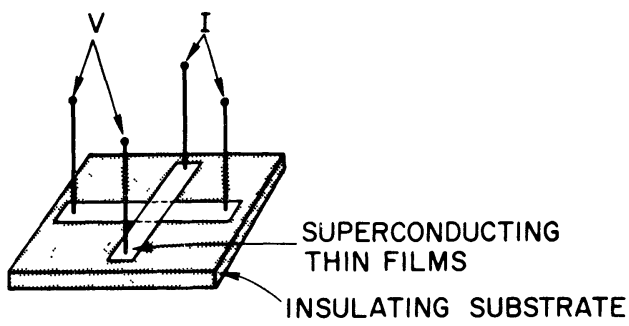

(a) THIN FILM JUNCTIONS

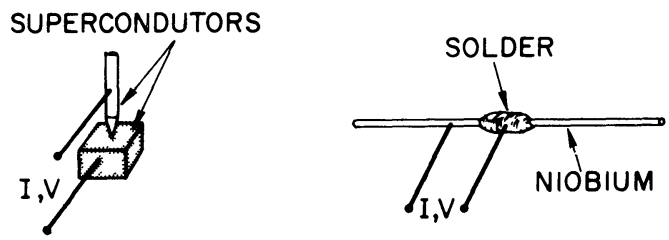

(b) POINT CONTACTS

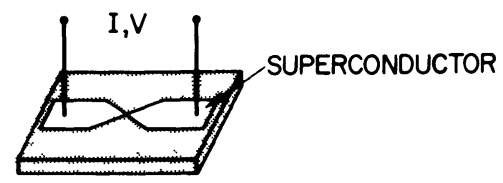

(c) THIN FILM BRIDGE

FIG. 8. - Different types of superconducting junctions. will discuss the operation of his "slug" interferometer [12] and give several examples of its use as a magnetometer and a galvanometer.

Recent work by Stewart [13] and by McCumber [14] on the current-voltage (I-V) relationship for various types of superconducting junctions has significant implications for magnetometer design. A schematic I-V curve for an evaporated film Josephson junction is shown on figure 9. First we note that the I-V relationship is directly related to the type of source used to drive the junction. For a constant current source, the type normally used in magnetometer circuits, we see that the voltage abruptly switches from zero to a rather large value when the

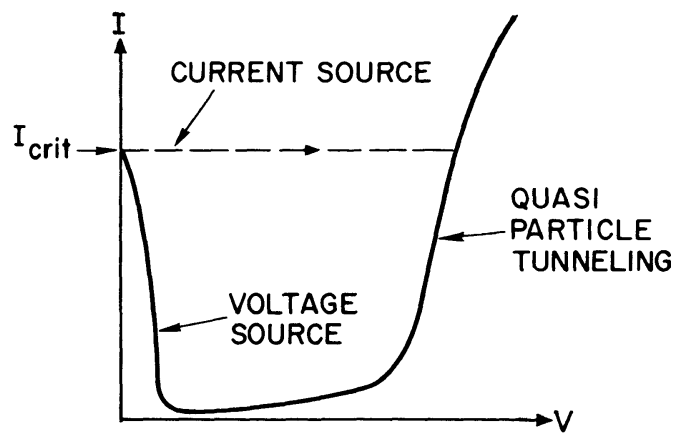

Fig. 9. - Current-voltage curve of thin film Josephson junction.

critical current is exceeded. For use as a magnetometer we want to measure the change in critical current with magnetic field, and a more optimum I-V curve would be one in which the voltage increased slowly, smoothly, and reversibly, from zero, once the critical current is exceeded. Stewart [13] and McCumber [14] have shown that the detailed nature of the I-V curve is related to the capacitance of the junction and the $a c$ Josephson effect frequency :

$$
\nu=\frac{2 e V}{h}
$$

where : $V=$ voltage drop across the junction, $h=$ Planck's constant.

Figure 10 shows several I-V curves for a junction driven by a constant voltage source. We see that

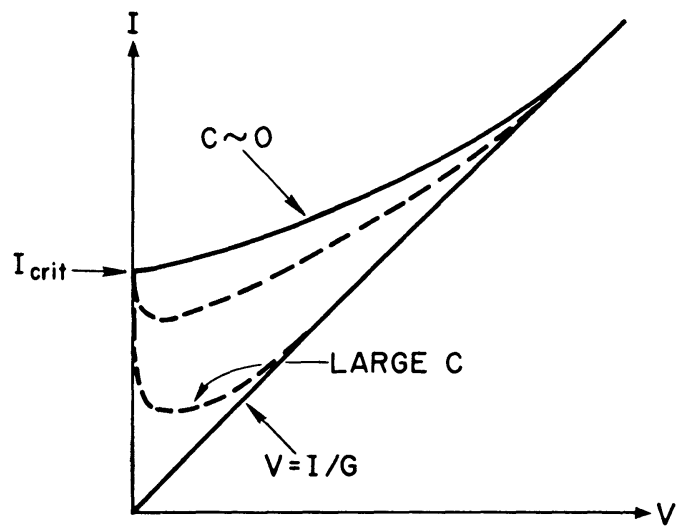

FIG. 10. - Current-voltage curves for a junction driven by a constant voltage source. 
TABLE I

SumMARY OF MAGNETOMETER PERFORMANGE

\begin{tabular}{|c|c|c|c|c|}
\hline MAGNETOMETER & $\begin{array}{l}\text { Resolution } \\
\text { (gauss) }\end{array}$ & $\begin{array}{c}\text { Time } \\
\text { CONSTANT } \\
(\mathrm{s})\end{array}$ & $\begin{array}{c}\text { VeGtor (V) } \\
\text { OR } \\
\text { Scalar (S) }\end{array}$ & $\sim \operatorname{SizE}(\mathrm{cm})$ \\
\hline - & - & - & - & - \\
\hline Meissner effect & $10^{-6}$ & 1 & V & $\begin{array}{c}\text { length } \sim 1 \\
\text { o.d. } \sim 0.2\end{array}$ \\
\hline Quantized flux & $10^{-7}$ & 1 & V & $\begin{array}{l}\text { length } \sim 1 \\
\text { o.d. } \sim 0.01\end{array}$ \\
\hline Flux flow & $10^{-10}$ & 1 & V & $\begin{array}{c}\text { length } \sim 1 \\
\text { o.d. } \sim 0.1\end{array}$ \\
\hline Double point contact & $7 \times 10^{-11}$ & 1 & V & loop dia. $\sim 1$ \\
\hline Glarke slug & $10^{-8}$ & 1 & V & loop dia. $\sim 2$ \\
\hline Gesium vapor & $10^{-7}$ & 1 & $\mathrm{~S}$ & $2 \mathrm{~cm}^{3}$ \\
\hline Proton precession & $10^{-6}$ & 1 & $\mathrm{~S}$ & $\begin{array}{c}\text { length } \sim 15 \\
\text { o.d. } \sim 15\end{array}$ \\
\hline Helium & $10^{-6}$ & 1 & V & $\begin{array}{c}\text { length } \sim 15 \\
\text { o.d. } \sim 15\end{array}$ \\
\hline Flux gate & $2 \times 10^{-6}$ & 1 & $\mathrm{~V}$ & $3 \mathrm{~cm}^{3}$ \\
\hline Rubidium vapor & $10^{-9}$ & 3 & $\mathrm{~V}$ & dia. $\sim 6$ \\
\hline
\end{tabular}

the more hysteretic curves correspond to junctions with large capacitance while small junction capacitance gives smooth nonhysteretic behavior.

The significant fact is that because point contact type junctions have very low capacity they should exhibit I-V curves more ideally suited for magnetometry applications. On the other hand, thin film evaporated junctions (dielectric barriers) have large capacity and exhibit hysteretic switching at the critical current. This type of junction is ideally suited for bistable circuit elements such as are useful in computers, but not for magnetometers.

Table I summarizes the reported magnetic field resolution of superconducting magnetometers and lists the most sensitive conventional (room temperature) instruments for comparison. The most sensitive superconducting magnetometer that we know of has been constructed on the basis of a double point contact interferometer as designed by Mercereau and his colleagues at the Ford Research Laboratory. Magnetic field resolution as good as $7 \times 10^{-11}$ gauss has been reported with this type of instrument (to be discussed by Mercereau in the following paper).

Gooling Technioues. - All known superconductors have critical temperatures near or below $20{ }^{\circ} \mathrm{K}$, and most of the superconducting magnetometers reported here have been operated at temperatures equal to the boiling point of liquid helium, about $4.2 \mathrm{o}$. In this temperature range a simple cooling technique of immersing the device in a liquid helium bath is adequate for providing the cryogenic environment. This part of the paper is concerned with the review of refrigeration techniques including passive liquid helium refill systems, solid cryogen systems, JouleThomson expansion cooling and miniature refrigerators.

Most research and development on superconducting magnetometers has been done in the laboratory by physicists and has generally been related to basic studies of the properties of the superconducting device. For this reason, the liquid helium refill system has been by far the most common technique used for cooling. These systems have varied from glass dewar vessels [15], vacuum insulated and liquid nitrogen shielded; to rather elaborate vessels without liquid nitrogen shielding that utilize the specific heat of the helium vapor for precooling leads and connections entering the liquid helium bath and also to cool radiation shrouds surrounding the dewar [16]. A carefully designed liquid helium dewar can have initial no-load boil-off rates lower than $1 \%$ per day. It can be nonmagnetic and very light.

The major disadvantage of systems like this for cooling magnetometers, particularly for field or space use, is associated with the liquid helium. The helium limits the orientation freedom of the dewar because the liquid cannot touch the warmer surface near the top of the dewar without causing excessive boil-off. Also, movement of this type tends to cause temperature gradients within the system that may affect the magnetometer sensitivity or resolution, or produce erroneous signals. In space applications, the separation of the liquid and gas phase presents further problems because of the zero $g$ environment. Another difficulty associated with liquid helium refill systems is the logistics of maintaining a supply of liquid helium near the test site and periodically refilling the dewar system. This can be especially difficult in field uses of the devices.

Cooling by solid cryogens has certain possibilities for superconducting devices. This technique was originally developed for cooling low temperature devices 
in a space environment where the phase separation problem mentioned earlier presents serious difficulties. For superconducting devices, solid hydrogen may provide an ideal temperature environment in that its triple point is $13.4 \mathrm{o}$ at a reduced pressure of 54 torrs, and the temperature can be further reduced to as low as $8{ }^{\circ} \mathrm{K}$ by very rapid pumping of the vapor away from the solid. Of course, to use even solid hydrogen cryogenic systems, we must develop superconducting magnetometers that will operate sufficiently well from $10-13 \mathrm{oK}$. As yet, very little effort has gone into studying devices in this range mainly because of the previously mentioned convenience of using liquid helium in the laboratory.

Solid cryogen systems have several distinct advantages over liquid helium refill systems and even over miniature refrigerators, especially for space flight. These advantages are : 1) No power is required in space; 2 ) The pumping is provided by opening a valve to the vacuum of space; 3 ) No moving parts except possibly a mechanism for controlling the valve opening and the resultant temperature of the solid cryogen; 4) It can easily be made nonmagnetic; 5) No problems are associated with the zero g environment because the cryogen is a solid and can be confined to a given region of the dewar; 6) The cooling is essentially vibration free and 7) The performance is not time-dependent, if proper heat-sinking is used, until all of the cryogen is gone.

There are also several disadvantages with this system : 1) It has poor standby capability, i.e., almost as much cryogen is used during standby as when the device is operating; 2) For non-space application, a vacuum pump is required to keep the pressure above the cryogen at the desired level for appropriate temperature control. This vacuum pump requires considerable amounts of power and it is normally magnetic.

The Aerojet General Corporation has developed a solid hydrogen system for temperatures from 10-12 $\mathrm{oK}$ with a total weight of about $150 \mathrm{lb}$ without pump and an 80-day run time. This is not a production refrigerator though.

The next technique suitable for cooling superconducting devices is an open-cycle Joule-Thomson expansion of a gas. Several commercial systems are available that utilize this technique; for example, Air Products Company has a liquid nitrogen cooled hydrogen-helium system with a total weight of 22 pounds that can absorb $0.5 \mathrm{~W}$ at $4.4 \mathrm{~K}$ and reach $3.6^{\circ} \mathrm{K}$ with no load. This system requires about 40-70 $\mathrm{mn}$ to reach operating temperature from room temperature and uses 0.7 liter/s of helium gas, $0.5 \mathrm{liter} / \mathrm{s}$ hydrogen gas, and 0.5 liter/hour of liquid nitrogen for continuous operation. This means that a continuous run-time of about 10 hours may be obtained from one standard $250-\mathrm{ft}^{3}$ cylinder each of helium and hydrogen. The advantages of the open-cycle Joule-Thomson system are : 1) It is relatively vibration free; 2 ) It can easily be made nonmagnetic and 3) No standby power is required if the cooling is fast enough so that operating temperatures can be reached when needed. The disadvantages are that rather large amounts of gaseous helium, hydrogen, and possibly nitrogen are needed for operation and these gases must be stored in high-pressure gas cylinders. Another disadvantage is that the JouleThomson expansion valve is very small and is likely to be plugged by impurities in the gases. Thus, very high purity and well-filtered gases are required. It is certainly feasible that this technique can be improved, as the market develops, to provide much more rapid cooling down - on the order of several minutes - with much lower gas requirements. This is particularly true when designed for cooling superconducting devices in which the device heat load is on the order of milliwatts.

The next cooling technique we will consider is the miniature mechanical refrigerator. It is my opinion that widespread field use of superconducting magnetometers depends on the availability of such refrigerators. Excellent reviews of refrigeration and liquifaction techniques are available in reference [18]; therefore, we will not go into detailed discussion of the thermodynamic cycles used in these refrigerators or of the various mechanical features. We do want to develop the general idea of the miniature refrigerator though, and emphasize such points as the size, weight, power requirement, thermal capacity, temperature, and price. We will also consider special requirements that need to be met for these refrigerators to be suitable for cooling superconducting magnetometers.

There have been, of course, many liquifiers constructed since the advent of the Collins machine in 1948, and great advances have been made with the large liquifiers, particularly systems with liquifaction capacities greater than 10 liters/hour of liquid helium. Turboexpanders seem to be leading this field, and machines with capacities up to several hundred liters/hour are now available. This is not the type of refrigerator suitable for cooling superconducting devices. These large machines weigh from a few thousand pounds to tens of thousands of pounds and are far too large for portable operation. Also, they are capable of absorbing heat loads from three to ten orders of magnitude larger than the loads produced by even the most inefficient superconducting magnetometer.

We are interested in refrigerators that are closedcycled, self-contained units that preferably weigh less than 100 pounds, require only line power for the operation, can reach and maintain temperatures well under $20^{\circ} \mathrm{K}$, are vibration free, and do not produce magnetic field noise or background at the superconducting magnetometer. Needless to say, a refrigerator meeting these specifications does not now exist. In fact, very few miniature refrigerators are available that will reach temperatures less than $20^{\circ} \mathrm{K}$ and these units were apparently not designed for use with sensitive magnetometers. Table II lists all of the small commercial refrigerators, to my knowledge, that will reach temperatures less than $20^{\circ} \mathrm{K}$. Several of these are not really miniature. Others are opencycle Joule-Thomson systems, and the weight of compressors or high pressure gas cylinders are not included in the indicated weight of these systems. The drastic spread in system weight and cost of refrigerators listed in this table indicates the potential of careful design directed toward optimizing these factors. This is especially true for cooling devices such as those to be used for superconducting magnetometers, where the heat loads are extremely small.

The closed-cycle mechanical systems that we are considering use helium gas as the working fluid and 
MANUFACTURER

Trade name
Model
Refrigeration range
Cycle

Closed-cycle?

Lowest temperature

Typical refrigeration

Cryostat dimensions

Compressor dimensions

Cryostat weight (1b)

Compressor weight (1b)

Mean time between fai-

lures (hr)

Maintenance interval (hr)

System cost
AIR Products

\begin{tabular}{cc}
\hline Cryo-tip & - \\
AC-3L & E-311 \\
$3.6-70^{\circ} \mathrm{K}$ & $3.3-4.5{ }^{\circ} \mathrm{K}$ \\
Joule-Thomson & Claude
\end{tabular}

Cryogenic TeChNology Inc.

\begin{tabular}{cc}
\hline Cryodyne & Cryodyne \\
$\# 400$ & 0110 \\
$3.8-4.5{ }^{\circ} \mathrm{K}$ & $6.5-25^{\circ} \mathrm{K}$ \\
Gifford-McMahon & Gifford-McMahon \\
with Joule- & \\
Thomson & \\
Yes & Yes
\end{tabular}

$3.8^{\circ} \mathrm{K}$

$1.2 \mathrm{~W}$ a $4.2^{\circ} \mathrm{K}$

18 in. dia.

48 in. long

$30 \times 32 \times 54$ in.

125

800

13,500

3,000

3,000

$\$ 40,000$

to 100,000

\section{$6.5{ }^{\circ} \mathrm{K}$}

$24 \times 12 \times 5$ in.

$21 \times 26 \times 41$ in.

30

175

13,500

3,000

$\$ 18,000$

Cryomech Inc.

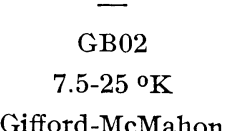

GB12

9-30 $\mathrm{K}$

Gifford-McMahon

Gifford-McMaho

Yes

$7.5^{\circ} \mathrm{K}$

$1 \mathrm{~W}$ a $10^{\circ} \mathrm{K}$

$5 \times 5 \times 21$ in.

$29 \times 19 \times 27$ in.

25

175

5,000

3,000

$\$ 13,200$
25

175

5,000

3,000

$\$ 13,200$

$\left(^{*}\right)$ From J. G. Daunt and W. S. Goree, Miniature Cryogenic Refrigerators, to be published.

are typically based on the Sterling cycle or GiffordMcMahon thermodynamic cycles [19]. These systems have a moving displacer piston located near the low temperature end of the refrigerator. This piston may cause magnetic field fluctuations, if magnetic, and vibration at the magnetometer. Alos, the temperature of these refrigerators varies at the displacer frequency, which is typically several hundred revolutions per minute, by as much as $\pm 1{ }^{\circ} \mathrm{K}$. Proper shock isolation and temperature control should allow minimization of these factors. Another significant factor, not given for most of the small refrigerators, is the required maintenance interval and the mean time between failures. From the available data [20], it is expected that maintenance intervals of no less than 1,000 hours and mean time between failures greater than 10,000 hours of continuous operation are possible.

Several companies are working on miniature turborefrigerators, and it appears possible that total turbosystems; with weights less than 100 pounds may be achieved with about $1 \mathrm{~W}$ capacity at $4 \mathrm{o}$. These turbosystems are all gas bearing systems and should be very reliable for long term operation. As of now, no performance data are available for refrigerators of this type. We can also note from Table I that there is a significant difference in weight, cost, and size of refrigerators operating at $8 \mathrm{oK}$ and those operating at about $4 \mathrm{oK}$. The Sterling and GiffordMcMahon machines use a regenerator in the cooling cycle, and the heat capacity of the regenerator material sets an intrinsic lower limit on the achievable temperature. To reach lower temperatures, a JouleThomson stage is usually used and this stage is precooled by the mechanical refrigerator at temperatures between $10-20 \mathrm{~K}$. The Joule-Thomson stage can add considerable weight and complexity to the system, especially if it is closed-cycle. This is due to the very stringent requirements on cleanliness of the helium gas in order to prevent plugging of the Joule-Thomson orifice.

\section{TABLE III}

Critical temperatures

OF SOME SUPERGONDUGTIVE MATERIALS SUITABLE FOR USE IN MAGNETOMETER CONSTRUGTION

\begin{tabular}{lc}
\multicolumn{1}{c}{ Material } & $\begin{array}{c}\text { CRITIGAl } \\
\text { TEMPERATURE }\end{array}$ \\
Indium & $\left({ }^{\circ} \mathrm{K}\right)$ \\
Tin & - \\
Tantalum & 3.4 \\
Lead & 3.7 \\
Niobium & 4.4 \\
Niobium-titanium & 7.2 \\
Niobium-zirconium & 9.2 \\
Niobium-nitride & $9-11$ \\
Niobium-tin & $10-11$ \\
& $14-15$ \\
& 18.0
\end{tabular}


[14] MCCumber (D. E.), J. Appl. Phys., 1968, 39, 3113.

[15] WhITE (G. K.), Experimental Techniques in LowTemperature Physics, Clarendon Press, Oxford, 1959.

[16] NiEndorf (L. R.) and Choksi (S. C.), Adv. in Cryogenic Engineering, K. D. Timmerhaus Ed., Plenum Press, N.Y., 1967, 286.

[17] Gross (U. E.) and FrIEdman (A. S.), "Investigation of Cryogenic-Solid Cooling Techniques", Aerojet-General Corporation, Azuza, Calif., ASDTDR-62-195, 1962.
[18] KROPSCHOT (R. H.), BIRMINGHAM (B. W.) and ManN (D. B.) Ed., Technology of Liquid Helium, National Bureau of Standards Monograph 111, N.B.S., Washington D.C., 1968.

[19] See Chap. 3 and 4, ref. [18] ; KöHLER (J. W. L.), Scientific American, 1965, 212, 119 ; and GrFFORD (W. E.), Adv. in Cryogenic Eng.; TrMMERhaus (K. D.), Ed., Plenum Press, 1966, 152.

[20] Daunt (J. D.) and GoreE (W. S.), Survey of Miniature Refrigerators, to be published.

[21] ROBERTS (B. W.), Superconductive Materials and some of their Properties, National Bureau of Standards, Technical Note-408, 1966. 